\title{
Intermolecular multiple scattering of electrons. II. Observed effects for $\mathbf{S F}_{6}$
}

\author{
Anding Jin ${ }^{\text {a) }}$ and Lawrence S. Bartell \\ Department of Chemistry, University of Michigan, Ann Arbor, Michigan 48109 \\ (Received 7 February 1983; accepted 11 March 1983)

\begin{abstract}
A theory of intermolecular multiple scattering of electrons by vapor molecules is tested by comparing predicted effects with effects observed over a wide range of sample densities. It is found that the theory, which contains no adjustable constants and is based on small angle approximations, gives a good account of experimental observations. The degree to which experimental structure refinements are degraded by multiple scattering is also examined. It is found that derived internuclear distances are disturbed very little even when the mean number of scatterings per electron is as high as 2 and the interference features are washed out by a factor of 2. Apparent amplitudes of vibration are influenced more significantly but are still correctable with fair precision.
\end{abstract}

\section{INTRODUCTION}

In a recent investigation of how a supersonic jet absorbs radiant energy and subsequently redistributes it among the various degrees of freedom, it was convenient to monitor the course of events by electron diffraction. ${ }^{1-3}$ Long-ignored questions concerning the validity of the method arose, however, that needed to be resolved. On the one hand, it was of interest to extend conditions into a collisional regime with vapor density exceeding that normally encountered in electron diffraction measurements. On the other, it was necessary to attain a precision in the determination of mean-square amplitudes of vibration substantially higher than that customarily sought. These conflicting aspects of the research prompted the present study of intermolecular multiple scattering.

In paper $\mathrm{I}^{4}$ of this series the theoretical problem was rendered tractable and reduced to explicit equations of simple form by introducing small-angle approximations. Whether the resultant rexpressions are of adequate validity in the 0 to 0.2 rad. region of scattering angle explored in the research on irradiated $S_{6}$ is tested experimentally in the following to gas densities far in excess of those encountered previously.

\section{EXPERIMENTAL}

\section{A. Apparatus and conditions}

The apparatus and sample of $\mathbf{S F}_{6}$ were identical with those of Ref. 1. Experimental conditions, listed in Table I, were also similar to those of the "control plates" in Refs. 1-3 except that an electron beam of lower current was used, for convenience, because the gas pressure was extended to higher values. Observed intensities are available as supplementary material. ${ }^{5}$

In addition to diffraction experiments, measurements were made of the gas throughput and the gas jet density profile as a function of reservoir pressure by procedures described in Refs. 1 and 2. Gas throughput measure-

\footnotetext{
2'Permanent address: Department of Chemistry, Nanjing Teachers College, Nanjing, 210024, People's Republic of China.
}

ments at the higher pressures were less precise than those at the lower pressures because of limitations in our apparatus. Presumably they are correct to within 10\%. Because experimental results agreed within experimental error with those calculated by a throughput algorithm described elsewhere, ${ }^{3}$ it was convenient to infer gas densities with the aid of the algorithm rather than by direct experiment. Hereafter in this paper, all calculated diffraction intensities are based on values of $\rho_{m}$ computed via the algorithm.

Measurements of the jet density profile, taken to be of form ${ }^{1,2}$

$$
\rho(z)=\rho_{m} /\left(1+b z^{2}\right)^{2}
$$

showed that $b$ increases (i.e., the jet becomes more diffuse) the higher the stagnation pressure. ${ }^{4}$ At lower pressures the flow is predominantly viscous and the jet is substantially narrower than a free jet produced by a thin-plate aperture. At higher pressures the flow becomes turbulent, sonically choked, and less dependent upon the nozzle length. The throughput and density distribution begin to tend toward those computed for thin-plate nozzles by Ashkenas and Sherman. ${ }^{6}$ Equations used in our treatment of jet density are in paper I. 4

TABLE I. Experimental conditions in diffraction experiments.

\begin{tabular}{lc} 
Nozzle to plate distances, mm & 210.77 \\
Diameter of hypodermic nozzle, mm & 0.12 \\
Length of hypodermic nozzle, mm & 5.6 \\
Nozzle-to-beam distance, mm & 0.41 \\
Sector (radius, mm) & $R^{3}(48)$ \\
Exposure time, s, and (pressure, Torr) & $4.3(500)$ \\
& $2.3(760)$ \\
& $2.0(1000)$ \\
& $1.0(2000)$ \\
& $0.73(3086)$ \\
Number of plates at each pressure & $0.61(3910)$ \\
Accelerating voltage, kV & $4^{2}$ \\
Beam current, A & 40 \\
\hline
\end{tabular}

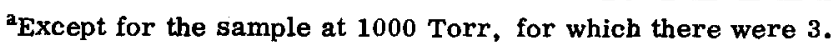




\section{B. Analysis of data}

The simplest and most practical way to analyze experimental diffraction intensities and to compare them with intensities calculated by the multiple scattering (IMS) method of paper $\mathrm{I}^{4}$ was to apply the standard procedures of structure determination. "That is, for reasons to be discussed in a later section, both the experimental intensities and the theoretical IMS intensities were analyzed by least squares comparisons with the standard model of single scattering. Derived parameters in each case were apparent bond lengths, apparent amplitudes of vibration, and the so-called index of resolution which can be thought of as a scaling parameter to characterize the degree of washing out of interference features by multiple scattering.

Least squares refinements were carried out without the imposition of geometric constraints to help absorb poorly understood radial distribution peak asymmetry effects. Asymmetry parameters $\hat{a}^{8}$ were chosen to be $2.1 \AA^{-1}$ for SF and FF (cis) and $1.2 \AA^{0-1}$ for FF(trans) distances, and $s^{2}$ weighting was adopted. Scattering factors were those of Schafer et al. ${ }^{3}$ and Tavard. ${ }^{10}$ Intramolecular multiple scattering corrections, ${ }^{11}$ while made in Refs. 1-3, were not included in analyses of experimental intensities reported in the following. Although they are straightforward at lower pressures, they are less so as pressure increases. Fortunately, their effect is modest in such a light molecule.

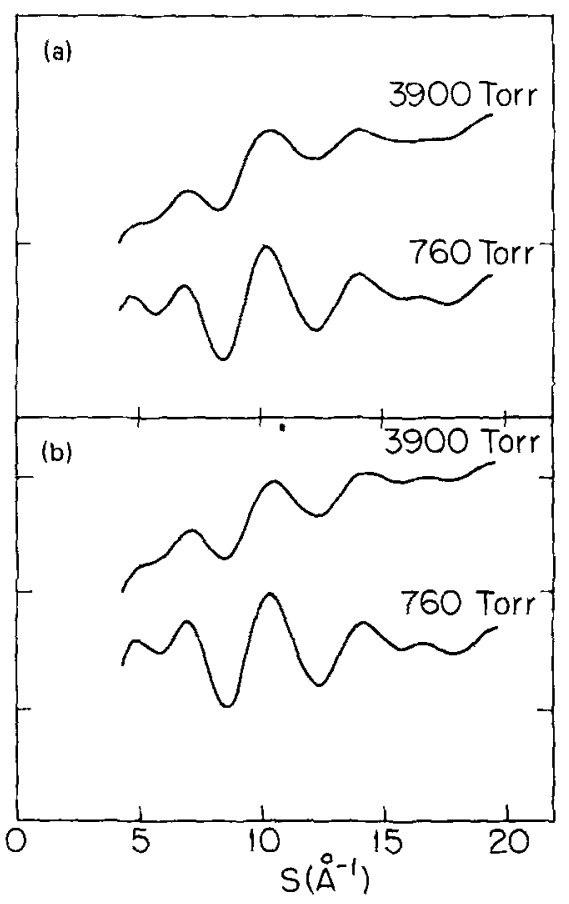

FIG. 1. Electron diffraction intensity curves corresponding to $\mathrm{SF}_{6}$ issuing from a nozzle $0.012 \mathrm{~cm}$ in diameter with stagnation pressures of 760 Torr (lower curve, each pair) and 3900 Torr (upper curve, each pair). Data have been "leveled" by dividing by the theoretical atomic intensities for single scattering, in each case. (a) Curves calculated by intermolecular multiple scattering theory of Ref. 4. (b) Experimental curves.

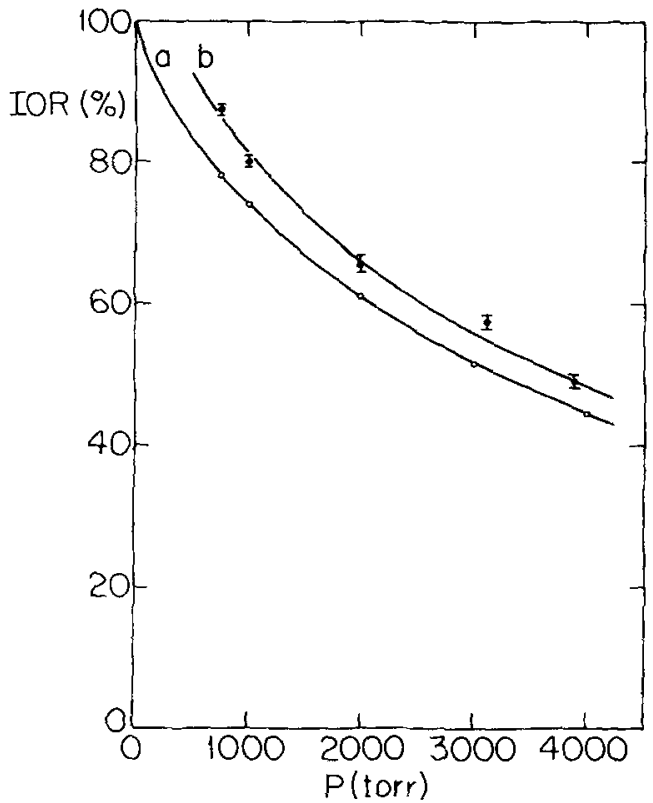

FIG. 2. (a) Theoretical (Ref. 4) and (b) experimental indices of resolution of reduced intensity curves of $\mathrm{SF}_{6}$ as a function of stagnation pressure. Error bars, $1 \sigma$, calculated as if residuals were random.

\section{RESULTS}

In principle, the most direct way to compare results of multiple scattering theory with experiment is to compare calculated intensities corresponding to various sample densities with experimental intensities. This is done in Fig. $l$ where, to render trends of the precipitously decreasing intensities most conspicuous, each curve is divided by the smooth theoretical atomic background curve calculated for single scattering. Data for 760 and 3900 Torr are shown. The effect of multiple scattering in washing out the interference features and in enhancing scattering at larger angles relative to smaller angles can be seen. It is more enlightening quantitatively, however, to treat the theoretical as well as the experimental points as if they were empirical data, and to analyze them by the standard least squares refinement procedure. This approach reduces large collections of points imparting mainly subjective impressions to single numbers suitable for quantitative comparisons. It also gives a very practical result at once, namely, the degree to which multiple scattering can degrade analyses of molecular parameters in diffraction studies of structure and vibrations.

Compared in Fig. 2 are the observed and IMS-calculated indices of resolution (IOR) plotted as functions of the sample reservoir pressure. Since the IOR is the "mean interference fringe visibility," or mean ratio of the observed to theoretical single scattered amplitudes of interference terms, the effect of multiple scattering in masking the molecular signal is apparent.

Because multiple scattering adds intensity disproportionately to signals at higher scattering angles, it has the effect of increasing the damping of the reduced intensity curve, $I_{\text {mol }} / I_{\text {bxgd }}$. Therefore, it causes ap- 


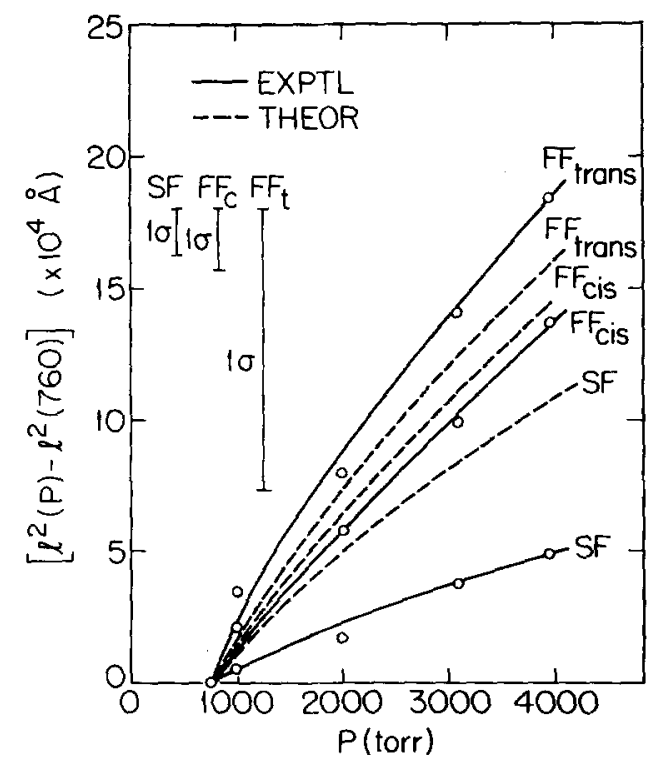

FIG. 3. Changes in apparent mean-square amplitudes of vibration of the atom pairs in $\mathrm{SF}_{6}$ as stagnation pressure is increased. Reference amplitudes are those for $1 \mathrm{~atm}$. Dashed curves, IMS theory of Ref. 4. Solid curves, experiment. Error bars, $1 \sigma$, calculated as if residuals were random.

parent mean-square amplitudes of vibration, $l^{2}$, derived from reduced intensity curves to be too large if the least-squares fitting model is that of single scattering. This is shown in Fig. 3 where least-squares analyses of observed and IMS-calculated curves are presented for the $S-F, F-F_{c i s}$, and $F-F_{\text {trans }}$ internuclear pairs of $\mathrm{SF}_{6}$. Analogously derived apparent internuclear distances are portrayed in Fig. 4.

Results in Figs, 1-4 were calculated through triple scattering with the aid of information in Tables $I$ and II of paper I, deleting the Gaussian term for $L_{0}$. This deletion corresponded to discarding the very small angle inelastic scattering which looks indistinguishable, in plots such as Figs. 1-4, from unscattered radiation. If the $L_{0}$ term had been retained, however, convergence of the fractions $f_{n}$ would only have been obtained at higher order scattering than triple and calculated differential cross sections of comparable quality would have been more tedious.

\section{DISCUSSION}

The effect of multiple scattering on the index of resolution is so large and systematic, as shown in Fig. 2 , that the IOR may serve as a useful measure of multiple scattering. Care must be exercised, however, because other experimental imperfections such as broadly delocalized sample ${ }^{12}$ and imprecise emulsion calibration constants ${ }^{13}$ can lead to similar effects. Such imperfections may be responsible for the offset of the experimental curve from the theoretical. Apparent meansquare amplitudes of vibration are influenced appreciably by multiple scattering also, as illustrated in Fig. 3, when analyses are based on refinements of the reduced molecular intensity $I_{\text {mol }} / I_{\text {bked }}$. If, as in some methods of refinement, the background had been $s u b-$ tracted from the theoretically leveled intensity ${ }^{3}$ instead of serving as a denominator, the effect of multiple scattering upon the apparent mean-square amplitude would have been much smaller.

Fortunately, the influence of multiple scattering upon internuclear distances derived via conventional refinements is very small as is evident in Fig. 4. A significant fraction of the effect of multiple scattering in Fig. 4 , an effect tending to make apparent distances increase as pressure increases, is in fact an artifact of the standard least-squares analysis having nothing to do with phase shifts of the molecular interference terms contributed by multiple scattering. As is well known, ${ }^{6}$ the single-scattering interference terms can be represented by a sinusoidal curve $\sin \left[s\left(r-\kappa s^{2}\right)\right]$ in which the frequency modulation coefficient $\kappa$ is related to an asymmetry parameter $\hat{a}$ expressing the skewness of the internuclear distance distribution, ${ }^{8}$ by

$$
\kappa=\hat{a} l^{4} / 6 \text {. }
$$

In least-squares refinements the $\hat{a}$ values were frozen as outlined in paper $I^{4}$ with $\hat{a}_{\mathrm{SF}}=\hat{a}_{\mathrm{FF}}=2.1 \AA^{-1}$ and $\hat{a}_{F F_{t}}$ $=1.2 \AA^{-1}$. Therefore, as sample pressure was increased, artifically enlarging the apparent mean-square amplitude $l^{2}$, the coefficient $\kappa$ was falsely inflated. This, in turn, led to a spuriously perturbed interatomic distance.

In diffraction investigations intended to produce precise molecular structures the present IMS theory could, in principle, be used to compensate for multiple scattering. A far better idea, however, is to avoid multiple scattering by keeping the average number of scatterings 4

$$
N_{t}=\sigma \int_{-\infty}^{\infty} \rho(z) d z
$$

much less than unity. In special cases, when electron diffraction is used to monitor processes taking place at such vapor densities that $N_{t}$ of Eq. (1) is not small com-

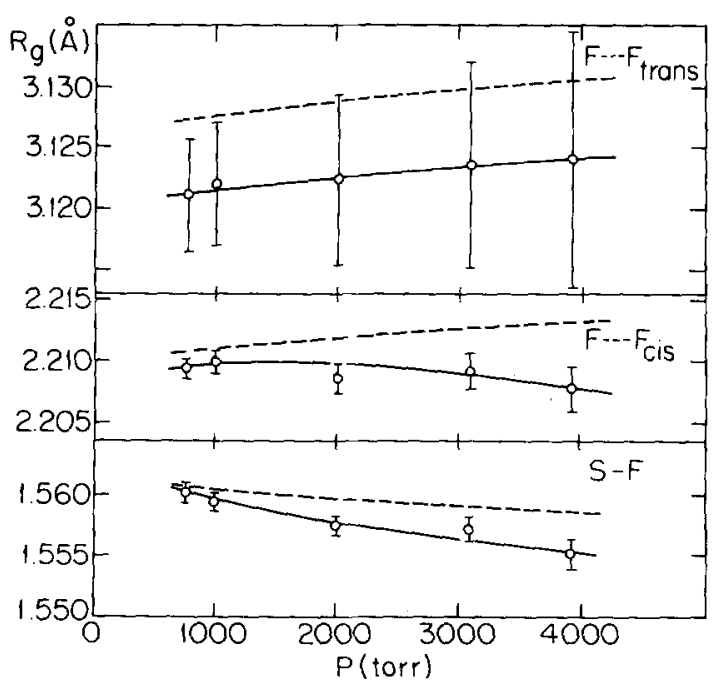

FIG. 4. Apparent mean internuclear distances for the atom pairs in $\mathrm{SF}_{6}$ found at various stagnation pressures. Dashed curves, IMS theory of Ref. 4. Solid curves, experiment. Error bars, $1 \sigma$, calculated as if residuals were random. 
pared with unity, it is advisable to run control plates taken under the same conditions of gas density. Despite the fact that electrons suffered, on average, about two scatterings at the higher pressures represented in Figs. 1-4 (see paper 1 ), the disturbance of derived molecular parameters was modest. Moreover, as explained in the foregoing, it could have been rendered even more modest if the standard refinement procedure had been modified slightly to cope with the unwanted background of multiple scattering.

Finally, it should be noted that the IMS theory derived in paper I on the basis of small angle approximations, is quite successful in accounting for the effects of multiple scattering displayed in Figs. 1-4. No adjustable parameters or empirically deduced scale factors were needed. The minor differences between observed and calculated quantities can be attributed, at least in part, to the severely truncated Gaussian sets chosen to represent the scattering factors, to the neglect of intramolecular (dynamic) multiple scattering, and to the adoption of the independent atom model. Prior observations ${ }^{1}$ and theoretical analyse ${ }^{14}$ have shown that, at low pressures, ${ }^{15}$ the major source of intensity residuals in the region of scattering angle considered in the present research is the redistribution of molecular electrons away from the spherical clouds characteristic of the independent atom model. If it were ever necessary to include the effect of bonding redistribution in IMS theory, it could be done within the framework of the present approach by the addition of Gaussians offset from nuclear positions.

We conclude that the problem of intermolecular multiple scattering in gas-phase electron diffraction can be effectively analyzed by the present approach. The simple, explicit expressions derived for double, triple, and higher scatterings lead to results in good agreement with observation.

\section{ACKNOWLEDGMENTS}

This research was supported by a grant from the National Science Foundation. We gratefully acknowledge a fellowship for one of us (A.J.) supported by the
People's Republic of China. We thank E. J. Valente, M. A. Kacner, and R. K. Heenan for their considerable assistance in carrying out this research. We are indebted to the University of Michigan Computing Center for a generous allocation of computing time.

${ }^{1}$ L. S. Bartell, M. A. Kacner, and S. R. Goates, J. Chem. Phys. 75, 2730, 2736 (1981).

${ }^{2}$ S. R. Goates, Ph. D. thesis, University of Michigan, 1981.

${ }^{3}$ M. A. Kacner, Ph.D. thesis, University of Michigan, 1983. ${ }^{4}$ L. S. Bartell and A. Jin, J. Chem. Phys. 78, 7159 (1983).

${ }^{5}$ See AIP document No. PAPS JCPSA-78-7165-9 for 9 pages of related materials including conditions under which the diffraction plates were taken, representative intensities and derived results. Order by PAPS number and journal reference from American Institute of Physics, Physics Auxiliary Publication Service, 335 E. 46 St., New York, NY 10017. The price is $\$ 1.50$ for each microfiche (98 pages), or $\$ 5$ for photocopies of up to 30 pages and $\$ 0.15$ for each page over 30 pages. Airmail is additional. Make checks payable to American Institute of Physics.

${ }^{6} \mathrm{~K}$. Ashkenas and F. S. Sherman, Rarified Gas Dynamics, Fourth Symposium, edited by J. M. de Leeuw (Academic, New York, 1966), Vol. II, p. 84.

${ }^{7}$ L. S. Bartell, in Physical Methods in Chemistry, 4th ed., edited by A. Weissberger and B. W. Rossiter (Interscience, New York, 1973).

${ }^{8}$ L. S. Bartell, J. Mol. Struct. 84, 117 (1982).

${ }^{9}$ K. L. Sellers, L. Schafer, and R. A. Bonham, J. Mol. Struct. 49, 125 (1978).

${ }^{10} \mathrm{C}$. Tavard, D. Nicoles, and M. Rouault, J. Chim. Phys. (Paris) 64, 540 (1967).

${ }^{11}$ See, for example, B. R. Miller and L. S. Bartell, J. Chem. Phys. 72, 800 (1980).

${ }^{12}$ L. S. Bartell, J. Appl. Phys. 31, 252 (1960).

${ }^{13}$ H. R. Foster, J. Appl. Phys. 41, 5344 (1970); L. S. Bartell and L. O. Brockway, ibid. 24, 656 (1953).

${ }^{14}$ P. Pulay, R. Mawhorter, D. A. Kohl, and M. Fink, J. Chem. Phys. (submitted); D. A. Kohl and L. S. Bartell, J. Chem. Phys. 51, 2891, 2896 (1969).

${ }^{15} \mathrm{At}$ higher pressures systematic intensity residuals are observed that arise because the effects of multiple scattering in experimental data are not compensated for in the single scattering fitting theory. These residuals are significantly correlated in form and position with those caused by the redistribution of molecular electrons. 